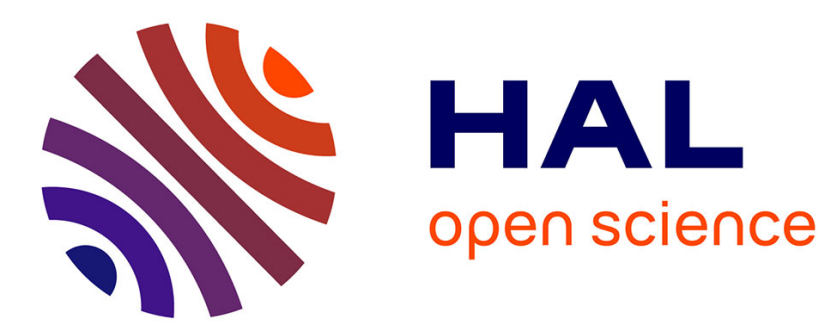

\title{
EQUILIBRIUM PHASES OF CHARGED COLLOIDS
}

D. Hone

\section{- To cite this version:}

D. Hone. EQUILIBRIUM PHASES OF CHARGED COLLOIDS. Journal de Physique Colloques, 1985, 46 (C3), pp.C3-21-C3-31. 10.1051/jphyscol:1985303 . jpa-00224620

\section{HAL Id: jpa-00224620 https://hal.science/jpa-00224620}

Submitted on 1 Jan 1985

HAL is a multi-disciplinary open access archive for the deposit and dissemination of scientific research documents, whether they are published or not. The documents may come from teaching and research institutions in France or abroad, or from public or private research centers.
L'archive ouverte pluridisciplinaire HAL, est destinée au dépôt et à la diffusion de documents scientifiques de niveau recherche, publiés ou non, émanant des établissements d'enseignement et de recherche français ou étrangers, des laboratoires publics ou privés. 


\section{Hone}

Department of Physics, University of Califormia, Santa Barbara, Califormia 93106, U.S.A.

Résumé - Nous passons en revue les thếories décrivant les phases cristallines et désordonnées des suspensions colloĩdales de sphères monodisperses de polystyrène. On teste un modèle d'interaction coulombienne écrantée (convenablement renormalisée) entre les particules. Les courbes théoriques de fusion et de transition de phase cristalline fcc - bcc sont présentées et comparées aux résultats expérimentaux en tenant compte de la dimension finie des sphères.

Abstract - We review the current theoretical picture of the crystalline and spatially disordered ("liquid") equilibrium phases of colloidal suspensions of monodisperse polystyrene spheres in polar fluids. The validity of a model of particles interacting through suitably renormalized screened coulomb interactions is explored. Theoretical predictions of the melting curve and fcc-bcc crystalline phase boundaries are presented. With the inclusion of corrections for finite sphere radius a satisfactory comparison is made between predicted and recent experimentally observed melting curves.

Among the most striking of the early observations made of colloidal suspensions of monodisperse polystyrene spheres was certainly the appearance, under suitable conditions, of the opalescence which signals the formation of crystallites. An obviously important question is: What values of the accessible parameters must be achieved to condense the solid from the disordered phase? But the disordered polyball "liquid" and the crystalline solid are not the only phases of interest. Direct microscopic observations have revealed(1) both fcc and bcc crystalline forms, and the conditions under which each of these constitutes the equilibrium phase is of equal interest. Of course, the explanation of the phase diagram of macroscopic systems is in general an important, complex, and fascinating problem. What is special about this sort of charged colloidal suspension is that the parameters, including average particle separation and interaction stiength, are readily adjustable experimentally over a wide range, and furthermore that the (electrostatic) interactions are well understood.

Whereas in ordinary atomic systems the phase boundaries are usually displayed in the pressure-temperature plane, or the liquid-solid coexistence region displayed in the temperature-inverse density plane, the convenient adjustable parameters for the colloidal suspension are: the polyball density, $\rho_{p}$, or equivalently the volume fraction $\Phi$ occupied by the polybalis ${ }^{p}$; the density of added salt, $\rho_{\text {salt, }}$ or the 
dimensionless screening effectiveness constant $k$ a where $k$ is the inverse Debye screening length for the electrostatic interactions and $a_{s}=\rho_{p}-1 / 3$
is the average polyball separation); and the dimensionless is the average poly

$$
\mathrm{g}^{2}=\frac{\mathrm{z}^{2} \mathrm{e}^{2}}{\varepsilon \mathrm{a}_{\mathrm{s}}} / \mathrm{k}_{\mathrm{B}} \mathrm{T},
$$

where $\mathrm{Ze}$ is the (negative) electric charge per polyball, $\varepsilon$ the static dielectric constant of the solvent (usually $\varepsilon \simeq 80$ for $\mathrm{H}_{2} 0$ at room temperature) and $T$ is the temperature. The few experimental melting curves have typically been displayed in the $\Phi, \rho_{\text {salt }}$ plane, and we will present results below in the plane whose coordinates are $\rho_{p}$ and ssalt, an essentialiy equivalent choice involving the two readily adjusted densities of polyballs and salt. On the other hand, the theory is most naturally expressed in terms of the variables $\mathrm{g}^{2}$ and $\mathrm{ka}_{\mathrm{s}} \cdot$ The $^{-}$ latter sets the range of the interaction relative to the average interparticle spacing, and therefore is a measure of the screening effectiveness. The former sets the only energy scale of the problem, the coulomb interaction between two polybalis at their average separation $a_{s}$, screened only by the dielectric constant $\varepsilon$ of the solvent. This energy is reduced to dimensionless form by division by $k_{B} T$, since that is how the energies in the problem enter the thermodynamic potentials (free energy) or partition function, but in contrast to the usual situation $T$ is not an interesting thermodynamic variable. It enters explicitly in $\mathrm{g}^{2}$ and in $\mathrm{ka}$ (see Eq.4 below) only in the product $\varepsilon \mathrm{T}$. As we have pointed out before ${ }^{(2)}$, the dielectric constant of the polar 1 iquid water would take the Curie form $\varepsilon=A / T$ where the permanent dipole moments of the water molecules completely free to rotate, making $\varepsilon T$ independent of temperature. In actuality the hindered rotation of the molecules leads to a product $\varepsilon T$ which varies by no more than $30 \%$ over the full accessible temperature range. The remaining $T$ dependences, of the polyball charge $z$ because of thermally induced dissociation and of the salt concentration because of ionization equilibrium, are weak. As a cautionary experimental note we should point out that probably the most temperature sensitive aspect of the system is the ion exchange resin which is frequently present to control the salt concentration; it readily pumps ions into the suspension with rising temperature.

A theory of the thermodynamic equilibrium phases of this system demands first a suitable model of the effective interaction between polybalis. As Hachisu $(3)$ has pointed out in the first paper of this conference, the DLVO potential associated with charged spheres in an electrolyte contains both attractive van der Waals and repulsive coulomb components; there is in addition the contact repulsion of the spheres themselves. In the solid phase, even at melting, the polyballs never approach one another close enough to sample the primary deep attractive minimum of the van der Waals potential. At larger separations the importance of the secondary attractive minimum increases with added salt concentration; for the range of parameters of interest to us here it is negligible not only with respect to $\mathrm{k}_{\mathrm{B}} \mathrm{T}$, but also with respect to the repulsive screened coulomb interaction, and it is only the latter which

we will take into consideration. not the first efforf in this direction. Among the earliest was a theory by Brenner 5 of melting via a Kirkwood-Alder transition of 
hard spheres, with an effective hard sphere radius, most appropriate presumably in the limit of strong screening and short range interactions (and where the secondary minimum due to van der Waals attraction remains unimportant). At the other limit of weak screening Marcelja, et al.(6) formulated a theory of melting for an effective one-component plasma along lines discussed at this conference by Hansen(7) (taking a critical value of the dimensionless coupling constant $\mathrm{g}^{2}$ or more conventionally $\Gamma$ for the plasma - as the melting condition). Hastings (8) has made use of Kirkwood's notion(9) of a density wave instability at freezing again to $100 \mathrm{k}$ at the liquid-solid transition, but he has used some rather crude approximations, notably to the direct correlation function which enters the theory (see the paper at this conference by Alexander and a paper in preparation(10) for a somewhat different approach to the freezing transition using Kirkwood's fundamental idea). Ohtsuki, et a1.(11) have carried out a cellular calculation of melting which requires suitable symmetric motions of nearest polyballs. The most recent publication is probably that of Shih and Stroud ( 12 ), on a Lindemann approach to freezing. Our own work in part also examines the melting curve, using a realistic interparticle potential over the full interesting range of screening effectiveness and particle density. But in addition we have predicted for the first time the boundary between the observed crystalline solid phases, bcc and $f(c$, within the same full parameter space. The thermal motion of the spheres has been included in the calculations, at least within an extended self-consistent harmonic approximation. Comparison with those experiments which exist with well characterized parameters has been gratifying, as we will show below.

With only electrostatic repulsion to be considered the appropriate underlying equation is the familiar Poisson-Boltzmann equation for the electrostatic potential

$$
-\nabla^{2} \phi(\vec{r})=\frac{4 \pi}{\varepsilon}\left[z e \rho_{p}(\vec{r})+\sum_{i}\left(\Omega_{i} z_{i} e\right) e^{-\beta_{i} z_{i}(\vec{r})}\right]
$$

where $\mathrm{th}_{i}$ and $z_{i}$ e are the number density (where $\phi(r)=0$ ) and charge of the $i^{\text {thi }}$ ionic species including the countercharges for the spheres; the huge size discrepancy between the ions and the polyballs allows the fluid to be treated as a charged continuum which can adjust adiabatically to the macroion motion. This highly non-linear equation not only requires numerical solution as it stands; it does not allow simple superposition of suitable solutions for single polyballs. However, one might hope to be valid the linearized form of the PoissonBoltzmann equation, namely the Debye-Hückel (hereafter, D-H) equation:

$$
\begin{gathered}
\left(\nabla^{2}-k^{2}\right) \phi \overrightarrow{(r)} \simeq \text { const. } \\
k^{2}=\frac{4 \pi e^{2}}{\varepsilon k_{B} T} \sum_{i} n_{i} z_{i}^{2},
\end{gathered}
$$

which does have the desirable features of simple analytic solutions and superposability. This approximation demands the smallness of the exponent $B z_{1} \mathrm{e}\left(\phi-\phi_{0}\right)$, where $\phi-\phi_{0}$ is the maximum potential difference to be found. An estimate of this in the present case, for polyballs of radius a and charge $\mathrm{Ze}$, is

$$
\frac{z_{i} e}{\varepsilon k_{B} T} \frac{z e}{\varepsilon a} \simeq \frac{7 z z_{i}}{a(A)}
$$


which is large $(\geqslant 1)$, even for the minimum $z_{i}=1$, for typical values of $Z$ of several hundred and $a \leqslant<1000 A$. Nevertheless, we have shown(13) that, as long as the charge $Z$ is suitably renormalized, the Debye-Hückel linearization can be used. The central idea is that in the solid, even near melting, the spheres remain sufficiently well separated that the potential gradients to which one is subjected due to any other (and therefore to all others) is sma11. Therefore, where a potential due to a polyball source is of interest, it is sufficiently slowly varying to be described by the linearized Debye-Hückel equation.

In order to determine the appropriate effective $\mathrm{D}$-H equation to be used let us consider the interactions in the perfect lattice, with no motion of the polyballs around the lattice sites. We divide the periodic crystal into identical Wigner-Seitz cells with a single polyball at the center of each and make the standard observation that the problem need only be solved within a single cell, the total solution being the periodic repetition of that one. The boundary condition is, by symmetry, that the electric field $\vec{E}=-\nabla \phi$ must vanish on the boundary of the cell. We make the usual approximate replacement of the geometrically complex cell by a sphere of the same volume (namely, $1 / \rho_{p}=a_{s}^{3}$ ). The spherically symmetric linearized problem now has the sblution

$$
\phi(r)=A \frac{e^{K r}}{r}+B \frac{e^{-K r}}{r}+\text { const. }
$$

The boundary condition $\phi^{\prime}(r=R)=0$, where the spherical cell radius $\mathrm{R}=(3 / 4 \pi)^{1 / 3} \mathrm{a}_{\mathrm{s}}$, sets one constant, and the arbitrary additive constant is convenientis taken to set the potential itself $\phi(r=R)=0$ there. How should the other constant be chosen? We could use the boundary condition at the polyball surface, but we are not particularly interested in matching the exact potential behavior there (indeed, matching that boundary condition results in a generally poor fit with the numerically integrated exact $P-B$ solution for small $r$ ). Instead we match the exact solution near $r=R$ more carefully, by insisting on the proper second derivative $\phi^{\prime \prime}(r=R)$ there; parenthetically, the third derivative is thereby also made correct. This not only establishes the parameters of the solution where the $\mathrm{D}-\mathrm{H}$ equation is expected to be most accurate. It also fixes the strength of the interaction, the centrally important quantity for the model, by matching a physical parameter directly related to these interactions- namely the osmotic pressyre or its volume derivative, the bulk modulus. It is readily shown (13) that the osmotic pressure is given by

$$
\Pi=-\left(\frac{\partial F}{\partial V}\right)_{N}=k_{B} T n(x)
$$

Conceptual1y, the pressure is simply due to the kinetic energy of a free gas of counterions of density $n(R)$ at the boundary, because the electrostatic pressure there vanishes by the condition $\vec{E}(R)=0$. Thus we must match the ionic density $n(R)$ at the boundary: but by Poisson's equation this is proportional to $\nabla 2 \phi(r)$, and the osmotic pressure is correctly given by properly matching $\phi^{\prime \prime}(r=R)$.

Now this solution to the D-H equation can be extrapolated back to the polyball radius; its slope there implies an effective charge $Z^{*}<Z$ to be used as a source for a medium described everywhere by the $\mathrm{D}-\mathrm{H}$ equation (see Fig. 1 for a representative plot of the renormalized $Z^{*} v S \mathrm{Z}$ ). In other words, we now have for the potential in the wigner-Seitz cel1 around the origin 


$$
\phi(r)=\frac{z^{\star} e^{2}}{\varepsilon}\left(\frac{e^{k a}}{1+k a}\right) \sum_{i}\left(\frac{e^{-k\left|\vec{r}-\vec{R}_{i}\right|}}{\left|\vec{r}-\vec{R}_{i}\right|}\right) \equiv \frac{1}{z} z^{\circ}\left(\vec{r}-\vec{R}_{j} \mid\right)
$$

where the sum is over all lattice sites except for the origin, and the screening wave vector $k$ is similarly determined by $z^{*}$ as

$$
\kappa^{2}=\frac{4 \pi e^{2}}{\varepsilon k_{B} T}\left[z \stackrel{*}{p} p^{+} \sum n_{i}{ }_{i}^{2}\right]
$$

with the sum only over the added salt ion species. The size dependent factor $e^{k a} /(1+k a)$ introduces yet another parameter into the problem. In what follows we will assume the polyball radius to be sufficiently small that we can replace this factor by unity. We will thus take as our model effectively a set of point particles of effective charge $z^{*}$ interacting pairwise through Yukawa potentials as described by Eq. (8). We note that the approximation of replacing the potential energy of a polyball by that of a point object of the same effective charge located at the actual polyball's center is well justified for $\mathrm{ka}<0.4$, as demonstrated by the "zero temperature" electrostatic boundary value calculation of Inoue and Wadati ${ }^{14)}$.

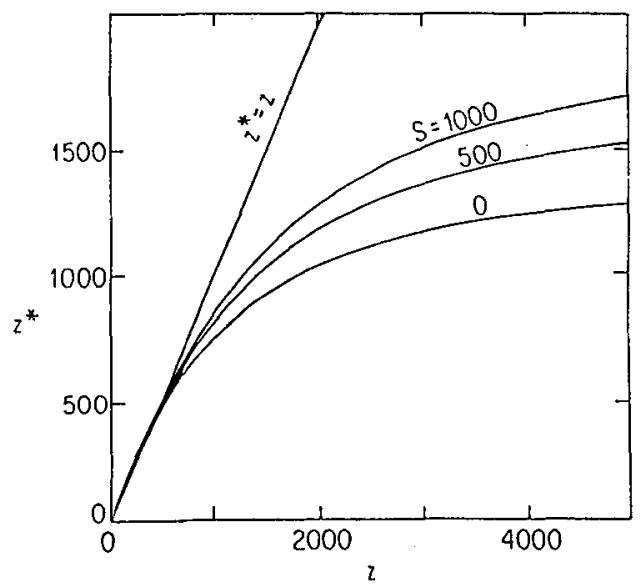

Fig. 1. Effective charge $Z^{*}$ as a function of actual charge $Z$ for several values of added electrolyte. S is the number of ion pairs per macroion.

It is essentially for this classical model of a system of point particles interacting through the potential (8) that we calculate the phase diagram below. Possible modifications necessary for comparison with experiment will be discussed later.

We know that both fcc and bcc crystals have been observed. Which is the most stable under given conditions? It is the one with the lowest free energy

$$
\Delta F \equiv F_{b c c}-F_{f c c}=\Delta E-T \Delta S,
$$


where, for simplicity, we look here only at single homogeneous phases and therefore compare free energies at fixed density, recognizing that the two-phase fcc-bcc coexistence region will be small. In this classical system momentum and velocity commute, and in the absence of velocity dependent forces kinetic and potential energy contributions to the free energy separate. Further, at room temperature we are far above the characterized eigen-frequencies of vibration: the Debye temperature $\theta_{\mathrm{D}} \sim \mathrm{k}_{\mathrm{D}} \sqrt{\mathrm{K} / \mathrm{m}}$, with the Debye wave vector $\mathrm{k}_{\mathrm{D}} \simeq 1 / \mathrm{a}_{\mathrm{S}}, \mathrm{K}$ a representative force constant and $M$ the polyball mass, so ${ }^{0}{ }_{\mathrm{D}} \sim 10^{-5} \mathrm{~K}$. The average kinetic energy is the classical value for each crystal lattice and the net contribution to $\Delta \mathrm{E}$ comes from the configurational energy difference

$$
\Delta E \simeq \frac{N}{2}\left(\frac{z^{2} e^{2}}{\varepsilon}\right) \Delta \sum_{i}<\frac{e^{-k r} i}{r_{i}}>,
$$

where the angle brackets denote a thermal average. In simplest approximation let us ignore thermal effects completely, taking $r_{i}=R_{i}$, the equilibrium lattice positions and neglecting the entropic contribution to the free energy difference. Then the sign of $\Delta E$, which determines the relative stability of fcc and bcc in this approximation, depends only on the single parameter $\mathrm{Ka}_{\mathrm{s}}$ :

$$
\Delta \mathrm{E} \propto \Delta \sum \frac{\exp \left(-\kappa a_{s} l_{i}\right)}{l_{i}}=\Delta \mathrm{E}\left(\kappa \mathrm{a}_{s}\right),
$$

where

$$
l_{i} \equiv R_{i} / a_{s}
$$

Qualitatively, we recognize that in the limit of no screening $\left(\kappa a_{s}=0\right)$ the coulamb, or classical Wigner, crystal takes the bcc form as most stable; in the opposite limit of large $\mathrm{ka}$ the rapid exponential falloff of interactions with distance implies that only the nearest neighbor terms matter, ensuring the stability of the close packed fcc structure, with the more distant (albeit more numerous) nearest neighbors. Indeed, in general one expects short range forces to favor fcc and long range ones to favor bcc. In the case of Yukawa interactions we find $(2)_{a}$ critical value of $k a_{S}=1.72$ where $\Delta \mathrm{E}=0$.

However, these energy differences $\Delta E$ are on $1 y$ of order one part in $10^{5}$ over a substantial range of values of $\mathrm{ka}$, and this extremely delicate balance demands the inclusion of the thermal effects we have heretofore neglected. Therefore we calculate directly the partition function

$$
\mathrm{e}^{-\mathrm{F} / \mathrm{k}_{\mathrm{B}} \mathrm{T}} \equiv \mathrm{z}=\mathrm{z}_{\mathrm{kin}} \mathrm{z}_{\mathrm{con}}
$$

where again the kinetic and configurational pieces factor in this classical system, and the free energy difference between the two structures is determined only by the latter:

$$
z_{\text {con }}=a_{s}{ }^{-3 N} \int d^{3} r_{i} \ldots d^{3} r_{N} \exp \left[-B V\left(\left\{\vec{r}_{i}\right\}\right)\right]
$$

where

$$
v\left(\left\{\vec{r}_{i}\right\}\right)=\sum v^{0}\left(\left|\vec{r}_{i}-\vec{r}_{j}\right|\right)
$$


and

$$
v^{\circ}(r)=\left(z^{*} e\right)^{2} \exp (-k r) / \varepsilon r
$$

as defined above in Eq. (8) in the limit of small $\mathrm{ka}$ (negligible polyball radius). The multiple integral of Eq. (15) can be made calculationally tractable, but still accurate, by adoption of a suitable mean field approximation - an extension of the self-consistent harmonic approximation(15) - in which a given polyball moves in the field of the others, whose own motion is taken into account on the average:

where

$$
v\left(\left\{\vec{x}_{i}\right\}\right)+\sum\left\langle v\left(\vec{x}_{i}\right)\right\rangle ; z_{\text {con }}=\zeta^{n}
$$

$$
\zeta=a_{s}{ }^{-3} \int d^{3} r \exp [-\beta<v(\vec{r})>]
$$

Here the mean field potential is given by

$$
\langle v(\vec{r})\rangle \int d^{3} x^{\prime}\left\langle p\left(\vec{r}^{\prime}\right)\right\rangle \quad v^{\circ}\left(\left|\vec{r}-\vec{r}^{\prime}\right|\right)
$$

and $\langle\rho(\vec{r})\rangle$, the average polyball density, is given approximately by a sum of gaussians about the lattice positions

$$
\left\langle\rho(\vec{r})>\simeq\left[\frac{3}{2 \pi<u^{2}>}\right]^{3 / 2} \sum_{i \neq 0} \exp \left[-\frac{3}{2<u^{2}}\right]\left|\vec{r}-\vec{R}_{i}\right|^{2}\right]
$$

The mean square excursion $\left\langle u^{2}\right\rangle$ is then to be calculated selfconsistently, for a polyball in the mean field potential $\langle\mathrm{v}(\overrightarrow{\mathrm{r}})\rangle$.

The detailed calculation has been done in Ref.(4). For any cubic crystal, the only case to be considered, symmetry imposes an expansion of $\langle\mathrm{v}(\overrightarrow{\mathrm{r}})\rangle$ in small $|\overrightarrow{\mathrm{r}}|$ containing only the Kubic Harmonics:



We have normalized to the natural energy scale, $g^{2} k_{B}{ }^{T}$. Then the several coefficients $V_{i}$ are functions only of $\mathrm{ka}$, the screening efficiency; $\kappa^{2}<\mathrm{u}^{2}>$, $i$ the dimensionless mean square excursion; and the lattice structure through the equilibrium lattice sums of Eq. (12) and suitable derivatives thereof. Similarly, the free energy per polyball can be written as

$$
\begin{aligned}
& \frac{F}{\bar{N}} \simeq-k_{B} T \text { In } \zeta-\frac{1}{2}\langle v(\vec{r})\rangle \\
& =\frac{1}{2} k_{B} T\left\{\tilde{B V} V_{0}-\frac{3}{2}+\frac{3}{4 \tilde{B} V_{2}}\left(\frac{5 V_{40}+3 V_{4 I}}{V_{2}}\right)+3 \ln \frac{\tilde{B} 2^{k{ }^{2} a_{S}^{2}}}{\pi}\right\}
\end{aligned}
$$

where the subtraction of half the average energy is necessitated, as usual, by the double counting inherent in all mean field theories, and the dimensionless temperature is again referred to the natural energy scale

$$
\tilde{\beta}=\frac{1}{\tilde{T}} \equiv \frac{z^{2} e^{2}}{\epsilon a_{s} k_{B}^{T}}
$$


Then $k^{2}<u^{2}>$, and thus the free energy difference of interest

$$
\frac{F(b c c)-F(f c c)}{k_{B} T}
$$

are functions only of $\widetilde{T}$ and $\mathrm{ka}_{\mathrm{S}}$, so that the fcc-bcc phase boundary predicted by this theory can be universally plotted in the $T, k_{s}$ plane. This has been done in Fig.2, which also includes the prêdicted melting curve. We note that at $\tilde{T}=T=0$ the fcc-bcc transition occurs at $\mathrm{ka}=1.72$, as was derived above. Thermal effects, both thermal vibrătion around lattice sites and entropic contributions, are responsible for all deviations of that boundary curve from a vertical line at this value of $\mathrm{ka}$. In particular, the bending over of the curve above $\mathrm{ka} \simeq 2.5$ is a purely entropic effect: The internal energies of esther lattice structure decreases exponentially with increasing $\mathrm{ka}$, because of the Yukawa form of the fundamental interaction, and ${ }^{\prime}$ therefore so does the difference in internal energies of fcc and bcc structures. On the other hand, the entropy (the final term in Eq. (23) is -TS) is a logarithm of one of these sums over Yukawa interactions for each lattice, and the difference is a logarithmic of the ratio of such sums. This increases monotonically for $\kappa a_{s}>1.72$, increasingly favoring bcc over $\mathrm{fcc}$, and it ultimately dominates the internal energy terms in the free energy difference. We also note that for sufficiently large $\tilde{T}$ (which basically means sufficiently small effective charge $Z$, since that is the principal adjustable parameter in T) the only stable crystalline phase is bcc, whatever the added salt concentration to change ka might be.

For the melting curve we should in principle again compare the relevant free energies - in this case for crystal and disordered fluid. That has effectively been the approach in the freezing theory Ref.(10) within a certain set of approximations. Instead, we start here from the solid side and invoke the widely successful semiempirical Lindemann criterion for the critical value of the mean square displacement $\left\langle u^{2}\right\rangle$, which gives a melting instability at

$$
\frac{\left.<\mathrm{u}^{2}\right\rangle}{\mathrm{a}_{\mathrm{s}}{ }^{2}}=\mathrm{C}
$$

with $C$ a constant in the very restricted range of about 0.025 and 0.033 for a broad range of potentials (16). Choosing the values of 0.025 and 0.030 as representative we have plotted the corresponding predicted melting curves as dashed lines in Fig.(2). It appears that, in accordance with predictions made(17) on the basis of a Landau theory of weakly first order phase transitions, the stable crystalline phase just below the melting point may always be bcc (of course, the limited quantitative reliability of the Lindemann estimate of melting and the closeness of the melting and $f c c-b c c$ boundary curves preclude a definite prediction, particularly at large $\kappa a_{s}$ ). We have obtained very similar numerical results from our theory of freezing. As we have already pointed out, the variables used for Fig. (2) may be the natural ones of the theory, but they are not appropriate for making comparison with experiment. As we have noted earlier (4), most of the range of values plotted in Fig.(2) is accessible with conventional values of polyball density $\rho_{p}$, effective charge $Z$ and salt concentration $\rho$ Let us consider in particular, the samples recently studied by $1 \mathrm{t}$. Lindsay and Chaikin ${ }^{18}$ with effective charge $Z=300$, and both $\mathrm{a}_{\mathrm{s}}=3.3 \times 10^{-5} \mathrm{~cm}$. and $\mathrm{a}_{\mathrm{s}}=1.9 \times 10^{-5} \mathrm{~cm}$. The corresponding values of $\mathrm{T}$ ar e $5 \times 10^{-3}$ and $3 \times 10^{-3}$ and in the absence of added sa1t the val- 


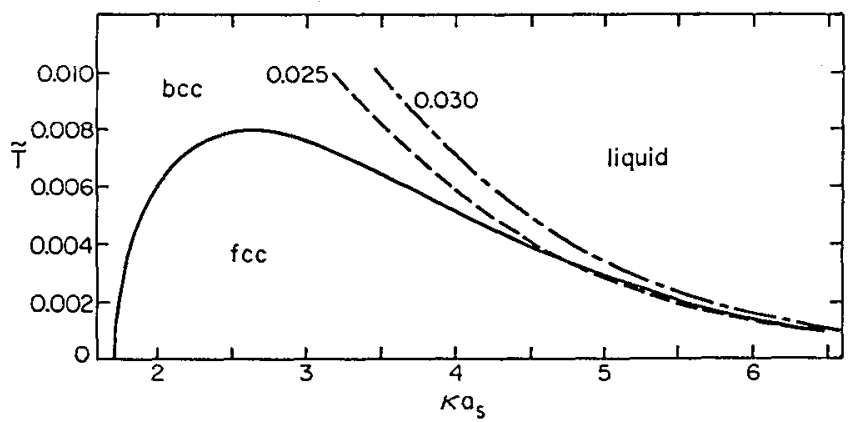

Fig. 2. The predicted phase diagram in the reduced temperature (see Eq.(24)), screening strength plane. The melting curves are labeled by values of the Lindemann parameter.

ues of $\mathrm{ka}$ are 2.8 and 3.7. In Fig.(3) we have replotted the phase diagram $s$ in the $p_{p}$, $\rho$, plane for this case of $z=300$, and we have indicated the experimental melting point value for the lower density. The agreement is excellent. Such would not be the case for a direct comparison between the high density experimental point ( $\rho_{\mathrm{salt}}=2.2 \times 10^{-4}$ molar at $\rho_{\mathrm{p}}=140 \times 10^{12} \mathrm{~cm}^{-3}$ ) and the $\mathrm{Z}=300$ theory. However, we recali that to pavoid an overabundance of parameters and to allow for universal plots of the type given in Fig. (2), we have theoretically dealt with an effective collection of point particles. The solution (8) to the Debye-Huickel equation, shows a finite size renormalization of the effective point charge

$$
\frac{z_{\text {eff }}}{z}=\frac{e^{k a}}{1+k a}
$$

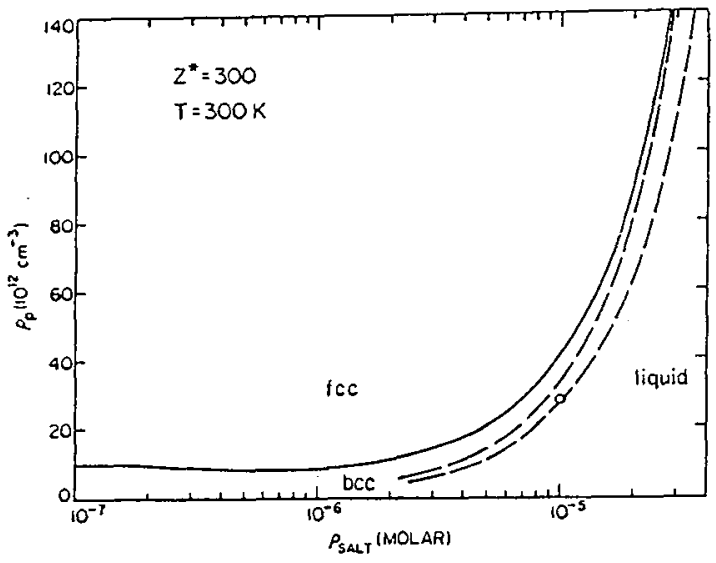

Fig. 3. Phase diagram in the polyball density $\left(\rho_{p}\right)$, added salt density plane for temperature $T=300 \mathrm{~K}$ and renormalized polyball charge of $Z^{*}=300$ (taken as having negligible radius: $k a \simeq 0$ ). The two dashed curves are the melting curves of Fig. 2 . 


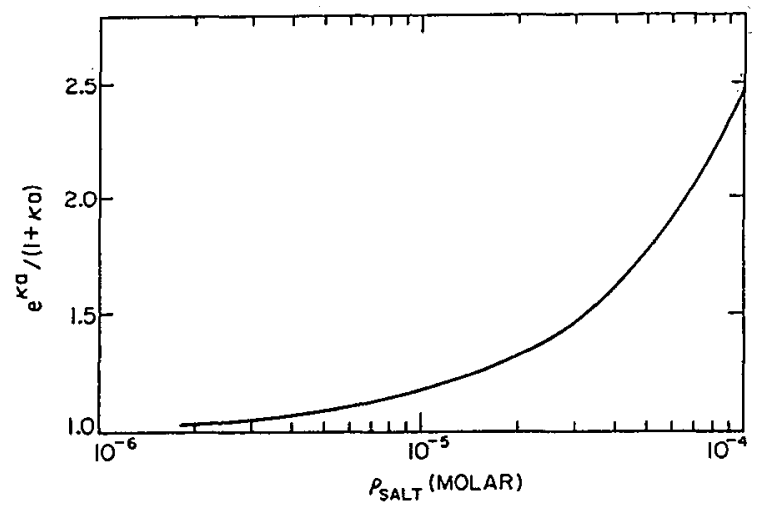

Fig. 4. The finite polyball size correction $Z_{f f} / Z=e^{k a} /(1+k a)$ along the upper melting curve in Fig. 2 f $(C=.0030)$ for $\mathrm{Z}=300$ and polybal1 radius $\mathrm{a}=545 \AA$.

This correction factor, plotted in Fig. (4) as a function of psalt (by using Eq. (9) for $Z=300$, and $\rho_{p}$ along the upper melting curve of Fig.2 at the appropriate value of $\mathrm{Ka}_{\mathrm{s}}$, and the experimental value of polyball radius $a=5.45 \times 10^{-6} \mathrm{~cm}$ ) becomes larger than 2.5 at the concentration $\rho, 210^{-4}$ molar where the dense experimental sample melts. The mefting curve is quite sensitive to the effective charge value, so we have incorporated the finite size correction into the melting curve prediction of Fig. (5). The agreement for polybali volume fraction $\Phi$ of $.01, .02$, and .03 is very satisfying, though the $\Phi=0.10$ point (the high density experiment at $a_{s}=1.9 \times 10^{-5}$ quoted above) still lies to the right of the theoretical



Fig. 5. The finite polyball size corrected melting curve for $z=300$, as predicted by the present theory (solid/curve) and by that of Ref.12 (short dashes for their Lindemann freezing theory and long dashes for their theory equating free energies of liquid and crystal). The experimental points (open circles) are from Ref.18. 
curve. We have not included the upward charge renormalization that added salt imposes(13), which would certainly improve the agreement. For comparison, we have also plotted in Fig. (5) the very recent predictions of melting by Shih and Stroud(12).

The agreement with those experiments which exist is gratifying, even though the theory is relatively crude. These are first order transitions, and under the usual experimental circumstances there will be a region of coexistence of two phases. The boundaries we have predicted will broaden into coexistence regions (which have, in fact, been observed in melting). This can in principle be calculated by properly comparing the free energies of competing phases at equal pressure so as to establish mechanical equilibrium rather than simply comparing the free energies of homogeneous phases at the same density. Nevertheless, we hope our predictions will stimulate experimental tests over a wide range of parameters.

\section{REFERENCES}

1. A. Kose, et al., J. Colloid Interface Sci.44, 330 (1973)

2. P. M. Chaikin, P. Pincus, S. Alexander and $\bar{D}$. Hone, J. Colloid Interface Sci. 89,555 (1982)

3. S. Hachisu (this issue)

4. D. Hone, S. Alexander, P. M. Chaikin and P. Pincus, J. Chem. Phys. $79,1474(1983)$

5. S. L. Brenner, J. Phys. Chem. 80, 1473 (1976)

6. S. Marcělja, D. J. Mitche11 and B. W. Ninham, Chem. Phys.Letters $43,353(1976)$

7. J.P. Hansen (this issue)

8. R. Hastings, J. Chem. Phys.68, 675 (1978)

9. J. Kirkwood, "Theory of Solutions" (Gordon and Breach, New York 1968).

10. S. Alexander (this issue); S. Alexander, P. M. Chaikin, D. Hone, P. Pincus and $D$. W. Schaefer (in preparation).

11. T. Ohtuski, A. Kishimoto, S. Mitaku and K. Okeno, Japan. J. App1. Phys. 20, 509 (1981)

12. W. - H. Shih and D. Stroud, J. Chem.Phys.79, 6254 (1983)

13. S. Alexander, et al., J. Chem. Phys. (in press).

14. M. Inoue and M. Wadati, J. Phys. Soc.Japan, 50, 1027 (1981)

15. N. S. Gillis, N. R. Werthamer, and T. R. Koehler, Phys. Rev.165, 951 (1968); H. Fukuyama and P. Platzman, Solid State Commun.15, 677 (1974)

16. J. P. Hansen and J. R. McDonald, "Theory of Simple Liquids" (Academic, New York,1976), p.361

17. S. Alexander and J. McTague, Phys.Rev.Letters 41, 702 (1978)

18. H. M. Lindsay and P. M. Chaikin, J. Chem. Phys.76, 3774 (1982) We are grateful to those authors for the unpublished more recent experimental values included in Fig. 5

19. See, e.g., S. Hachisu, Y. Kobayashi and A. Kose, J. Colloid Interface Sci. $\underline{42}, 342$ (1973). 